\title{
The role of exercise in the management of adverse effects of androgen deprivation therapy for prostate cancer: a rapid review
}

Kim Edmunds ${ }^{1,2}$ ORCID 0000-0001-7379-1294

Haitham Tuffaha ${ }^{3}$ ORCID 0000-0003-2993-3480

Paul Scuffham ${ }^{1,2}$ ORCID 0000-0001-5931-642X

Daniel A Galvão ${ }^{4}$ ORCID 0000-0002-8209-2281

Robert U Newton ${ }^{4,5}$ ORCID 0000-0003-0302-6129

${ }^{1}$ Centre for Applied Health Economics, Griffith University, Nathan, Australia.

${ }^{2}$ Menzies Health Institute Queensland, Griffith University, Gold Coast, Australia.

${ }^{3}$ Centre for the Business and Economics of Health, University of Queensland, Brisbane, Australia.

${ }^{4}$ Exercise Medicine Research Institute, Edith Cowan University, Joondalup, Australia.

${ }^{5}$ School of Human Movement and Nutrition Sciences, University of Queensland, Brisbane, Australia.

Corresponding author*

Ek.edmunds@griffith.edu.au

T 61737359112

Key words: prostate cancer, androgen deprivation therapy, adverse effects, management, exercise medicine 


\section{Abstract}

Purpose

Prostate cancer (PCa) is the most commonly diagnosed cancer in Australia, accounting for one quarter of all new cancer diagnoses for males. Androgen deprivation therapy (ADT) is the standard first-line therapy for metastatic PCa but is also used across much of the spectrum of disease. Unfortunately, debilitating adverse effects are a significant and largely unavoidable feature of ADT. A recent systematic review of adverse effects of ADT identified 19 sub-groups classified according to the Common Terminology Criteria for Adverse Events (CTCAE) Version 5.0. The potential for multiple simultaneous adverse effects, their associated management and the impact of adverse effects on cancer outcomes and quality of life are important considerations in the treatment and supportive care of men with PCa. Exercise is increasingly being recognized as an efficacious strategy in managing these adverse effects.

Methods

A rapid review was undertaken to examine the role of exercise in the management of the most commonly reported ADT adverse effects classified according to the CTCAE sub-groups. A systematic search was conducted in Medline, PsycINFO, Google Scholar and Google for the years 2010 to September 2019 to identify the benefits of exercise in managing the adverse effects of ADT for PCa.

Results

There is strong evidence for exercise as medicine in addressing several of the adverse effects of PCa such as loss of muscle mass and strength, fatigue and declining physical function. Moderate level evidence for PCa exists for exercise induced improvements in depression and anxiety, bone loss and sexual dysfunction. While evidence of the effectiveness of exercise is lacking for many adverse effects of ADT for PCa, evidence in the cancer population as a whole or other clinical populations is strong, and many clinical guidelines recommend exercise as a fundamental part of their clinical management. With the exception of gynaecomastia and breast pain, there is increasing evidence ( $\mathrm{PCa}$, cancer or other clinical populations) to suggest that exercise has the potential to reduce and even prevent many of the adverse effects of ADT, thus improving survivorship outcomes for men with PCa.

Conclusion

Exercise has the potential to reduce and even prevent many of the adverse effects of ADT, thus improving survivorship outcomes for men with PCa. The use of exercise for PCa management has the potential to translate into health and economic benefits in improved quality of life and fewer complications, resulting in savings to the health care system, enhanced productivity and reduced patient and carer burden. Exercise thus has the potential to improve quality of life for this population as well as generate significant cost savings. 


\section{Introduction}

2 Prostate cancer (PCa) is the most commonly diagnosed cancer in Australian men, accounting for one quarter of

3 all new cancer diagnoses for males [1]. With a predicted 5-year global prevalence of 3.7 million in 2018 and an

4 incidence of 18,274 in Australia alone, PCa represents a considerable public health burden [2]. The cost of PCa

5 in Australia has been estimated at US\$270.9 million in 2016, rising to US\$384.3 million by 2025 [3]. Androgen

6 deprivation therapy (ADT) is the standard first-line therapy for metastatic PCa but also improves survival in

7 men with high-risk localised, locally advanced and castrate resistant PCa. Thus, ADT is used across much of the

8 spectrum of disease, often for considerable periods of time [4]. Debilitating adverse effects are a significant and

9 largely unavoidable feature of ADT for men with PCa. A recent systematic review [5] identified 19 adverse

10 effect sub-groups classified according to the Common Terminology Criteria for Adverse Events (CTCAE)

11 Version 5.0 [6]. Statistically significant increased risks were evident for all nine ADT adverse effect groups

12 (musculoskeletal, metabolic, cardiac, nervous system, vascular, hepatobiliary, reproductive, psychiatric and

13 general disorders), with evidence of increased risks for 17 out of the 19 sub-groups [5]. Cognitive disorder and

14 dementia were the exceptions, with inconclusive results or suggested associations with ADT only, based on

15 currently available evidence[5]. Given the incidence of these adverse effects, there is a need for management

16 strategies that minimise the burden of PCa treatment with ADT. The potential for multiple simultaneous adverse

17 effects, the impact these have on cancer outcomes and quality of life, as well as their associated management,

18 are important considerations in the treatment and supportive care of men with PCa. While the value of exercise

19 as medicine has long been acknowledged for the general population [7] as well as cancer [8, 9] and PCa

20 populations $[10,11]$, it is increasingly being recognized as an efficacious strategy in managing the adverse

21 effects associated with cancer treatment.

22 In 2019, the Exercise and Sports Science Australia (ESSA) position statement and the American College of

23 Sports Medicine (ACSM) guidelines were updated with current scientific evidence, clinical experience and

24 exercise science principles. They emphasise the importance of an appropriate exercise prescription for cancer patients, which is individualised and targeted for the specific health issues most impacting the patient [12, 13]. The ACSM guidelines for cancer survivors recommend moderate intensity aerobic training at least three times per week, for at least 30 minutes. The addition of resistance exercise to aerobic training, at least twice a week, using at least two sets of eight to 15 repetitions, results in similar benefits, though evidence suggests resistance training alone may not be enough [13]. The ESSA guidelines recommend a more tailored approach with exercise mode and dosage prescribed specifically to ameliorate, in priority order, the health issues and mortality risks of greatest concern for the patient. Both these documents were based on extensive evidence reviews to ascertain the strength of evidence supporting the use of exercise for cancer patients and survivors. Strong evidence was available for a number of cancer-related outcomes: anxiety and depression, fatigue, health related quality of life (HRQoL) and physical function. Moderate evidence was also available for bone health and sleep. While organisations such as the American Society of Clinical Oncology (ASCO) [14], the National Comprehensive Cancer Network [15] and the National Institute for Care and Excellence[16] have PCa survivorship guidelines that support exercise for adverse effect management, there is no comprehensive review of the benefits of exercise in managing the adverse effects of ADT for PCa. Therefore, the aim of this review is to identify existing evidence of the benefits of exercise in managing the adverse effects of ADT for PCa. 
1 A rapid review of the literature was undertaken by the authors to examine the role of exercise in the

2 management of ADT adverse effects outlined above [5]. A search was conducted in Medline, PsycINFO,

3 Google Scholar and Google for the years 2010 to September 2019. A period of ten years of evidence generation

4 was chosen by the research team in order to focus on more current treatment regimes and approaches to management. Search terms comprised: androgen deprivation; prostate cancer; adverse effects; adverse events;

6 toxicity; complications; management; guidelines; and exercise; or physical activity. The Population,

7 Intervention, Comparator and Outcome (PICO) inclusion and exclusion criteria are outlined in Table 1.

8 Evidence was prioritised according to the Oxford Centre for Evidence-based Medicine (OECBM) 2011 Levels

9 of Evidence [17] and included: A. systematic reviews; and B. analytic studies, such as randomised controlled

10 trials (RCTs). Where little or no evidence of the effect of exercise was available for men with PCa receiving

11 ADT for a particular adverse effect, a subsequent search was conducted to identify evidence of exercise impact

12 for other cancer patients, disease specific evidence (e.g. diabetes or cardiovascular disorders) or evidence from

13 the general population. This required removing prostate and androgen deprivation from the search terms and

14 including other terms relevant to ADT adverse effects like hormone therapy, cardiovascular, diabetes,

15 metabolic, depression, falls, fractures, cognitive, dementia, hot flushes, deep vein thrombosis and liver disease.

16 PICO inclusion criteria were also broadened. The population parameter included the general population and

17 other disease risks; the intervention parameter other adverse effects or disease risks; and the outcomes parameter

18 adverse effect or disease risk management. The comparator parameter was maintained. PICO exclusion criteria

19 remained the same with the exception of the population parameter which was changed to younger people $(<50$

20 years). Qualitative judgments of currently existing evidence were based on agreement between authors due to

21 the heterogeneity of sources and paucity of evidence in some areas.

\section{Table 1. PICO inclusion and exclusion criteria}

\begin{tabular}{|l|l|l|}
\hline Parameter & Inclusion criteria & Exclusion criteria \\
\hline Population & $\begin{array}{l}\text { Men with PCa receiving androgen } \\
\text { deprivation therapy ADT }\end{array}$ & PCa population not receiving ADT \\
\hline Intervention & $\begin{array}{l}\text { Supervised or prescribed exercise to } \\
\text { manage ADT adverse effects for PCa }\end{array}$ & $\begin{array}{l}\text { Unsupervised or purely recreational } \\
\text { exercise (i.e. without prescription or } \\
\text { professional oversight) }\end{array}$ \\
\hline Comparator & $\begin{array}{l}\text { No management, pharmaceuticals or } \\
\text { medical treatment only }\end{array}$ & - \\
\hline Outcomes & $\begin{array}{l}\text { ADT adverse effect management } \\
\text { (higher levels of evidence: } \\
\text { Systematic Review, Meta-analysis, } \\
\text { RCT, cohort study, population-based } \\
\text { observational study) }\end{array}$ & $\begin{array}{l}\text { Lower ranked evidence (e.g. review, } \\
\text { cross sectional study) }\end{array}$ \\
\hline
\end{tabular}

Key: ADT androgen deprivation therapy; PCa prostate cancer; RCT randomised controlled trial

24 The adverse effects of ADT were classified according to the CTCAE [6] sub-groups to facilitate comparison of available evidence for specific adverse effects [5]. Evidence of the role of exercise in addressing each of these adverse effect sub-groups is described below.

\section{Results}

28 Results will be presented in two sections. Section 1 will present the evidence for the role of exercise in managing the adverse effects of ADT. Section 2 will provide evidence from other populations for the role of exercise in managing other adverse effects or disease risks, where there is little or no evidence for the PCa

31 population. 
1 1. Evidence of the effectiveness of exercise in managing the adverse effects of ADT

2 Musculoskeletal changes

3 Bone loss, osteoporosis and fracture risk

4 Management of bone loss, osteoporosis and fracture risk consists of baseline assessment of bone mineral density

5 (BMD) using dual-energy X-ray absorptiometry (DXA) of the spine, hips and forearm, which should then be

6 followed by lifestyle interventions comprising exercise and diet, and pharmacological treatment with

7 bisphosphonates only if required [18]. Resistance exercise training and high impact loading exercises help to

8 mitigate ADT related bone loss, thus improving bone health and reducing fracture risk. While one systematic

9 review and meta-analysis of the most effective methods for preventing osteoporosis in men taking ADT for PCa

10 concluded exercise alone was insufficient to address bone loss[19], improvements in or preservation of BMD

11 for men with PCa receiving ADT who participated in an exercise intervention have been reported in three recent

12 RCTs [20-22]. These studies reported improvements in hip and/or spine BMD (where the most problematic

13 fractures occur). Endurance and resistance exercise improved bone mineral density in the right and left total hip

14 and right and left femur in one study [21], combined resistance, aerobic and impact loading exercise improved

15 lumbar spine BMD in another study [20], and combined impact loading and resistance exercise attenuated the

16 decline in both spine and hip BMD in another [22]. These findings highlight the impact of targeted exercise and

17 are critical for a population at high risk of falls and fractures [5].

18 Metabolic changes

19 Body composition

20 Androgen deprivation therapy causes increased fat mass and decreased lean mass possibly progressing to

21 sarcopenic obesity in many men with PCa. Increased abdominal fat promotes insulin resistance and reduced lean mass contributes by reducing glucose uptake in muscles. Visceral obesity has also been associated with increased fatigue [23], reductions in BMD and bone strength and the potential, if not managed, to impact adversely on other disease risks, morbidity and mortality. An intensive lifestyle intervention should be instituted to prevent weight gain and worsening insulin resistance [18]. Exercise has been shown to be effective in improving body composition in seven systematic reviews [24-30]. Outside these systematic reviews, evidence of exercise induced reductions in fat mass were reported in one RCT [31], and evidence for increased lean mass and/or muscle strength in four RCTs [20, 22, 32, 33]. gain (increased waist circumference), hyperglycemia (increased fasting glucose), hypertriglyceridemia (increased triglycerides), decreased serum high density lipoprotein (HDL), increased insulin and hypertension. Importantly, close monitoring and intervention is recommended, particularly in the first year of ADT, because adverse effects can occur from three months post treatment $[34,35]$ and there are subsequent risks associated with diabetes and cardiovascular health. Exercise and lifestyle change are important considerations in addressing these risks. Results from one systematic review on the effect of exercise for men receiving ADT reported inconclusive results for cardiometabolic risk markers [25].

Diabetes

Diabetes risk is a serious concern for PCa survivors receiving ADT due to the effect of ADT on insulin sensitivity and other cardiovascular disease (CVD) risk factors. Exercise is recognised as a critical tool in the prevention and treatment of diabetes [36-38], however, no PCa specific evidence exists in relation to the effect of exercise in mitigating diabetes. 
3 Androgen deprivation therapy is associated with elevated cardiovascular (CV) morbidity or mortality, and given

4 the aforementioned metabolic effects of ADT and associated CV complications, it is advisable that patients

5 receiving ADT undergo metabolic evaluation at baseline and periodically during follow-up visits [39]. There is,

6 however, a paucity of consistent data on the impact of exercise on these outcomes for men receiving ADT for

7 PCa. Only one systematic review included evidence in relation to PCa survivors, which showed exercise

8 training is associated with significant improvements in vascular endothelial function and peak oxygen volume

$9\left(\mathrm{VO}_{2}\right)$ [40]. Improvements in flow-mediated dilation (FMD) are associated with improved CVD risk

10 independently of more traditional risk factors such as body mass index (BMI), cholesterol or blood pressure

11 [40]. The effect of exercise on change in FMD (1.3\%) was similar to that reported for other healthy and diseased 12 populations [40].

\section{Nervous system disorders}

\section{Cognitive impairment and dementia}

15 While the suppression or blocking of testosterone by ADT is likely to increase cognitive decline, there is a lack

16 of conclusive evidence in relation to the deleterious effect of ADT on cognition, especially verbal, spatial and

17 executive functioning; thus, there is no definitive recommendation for preventing or treating cognitive

18 impairment in men with PCa [5]. Age, stage of disease and co-morbidities may contribute to cognitive changes

19 in patients on long-term ADT [41]. While evidence exists to show that exercise improves cognitive function in

20 the general population, this has not been demonstrated in PCa patients receiving ADT.

$21 \quad$ Stroke

22 Little evidence exists in relation to the impact of exercise in reducing risk of stroke or its management

23 specifically for men with PCa receiving ADT outside improved endothelial function as for cardiovascular

24 disorders above [40].

\section{Vascular disorders}

\section{Hypertension}

27 Androgen deprivation therapy, particularly second generation hormonal agents such as abiraterone acetate and enzalutamide, is associated with significant increases in risk of hypertension [5]. While there is currently no evidence of the impact of exercise on hypertension for men with PCa receiving ADT, regular screening and lifestyle modification (including exercise and diet) are recommended [18].

\section{Thromboembolic events}

While there is evidence of an association between ADT and increased risk of thromboembolic events such as deep vein thrombosis (DVT) and pulmonary embolus (PE) [5], no PCa specific evidence currently exists for the role of exercise in mitigating this effect.

Hot flashes There is an established association between ADT and vasomotor flushing across various treatments and stages of PCa from locally advanced to metastatic castration resistant prostate cancer (mCRPC) [5]. Exercise could potentially benefit men with PCa receiving ADT and experiencing hot flashes, however, no evidence currently exists, and more research is needed. increased risk of hepatotoxicity [5]. Currently, there is no evidence for exercise as a mitigating strategy 
1 Reproductive system disorders

\section{Gynaecomastia and breast pain}

3 Gynaecomastia and breast pain or mastalgia, often referred to in the literature as breast events, are a common

4 adverse effect of non-steroidal antiandrogen therapy (bicalutamide, flutamide) and, while less common for

5 GnRH agonist therapy, can seriously impact men's masculinity and quality of life [42]. There is no evidence to

6 support the use of exercise as a strategy to manage gynaecomastia in men with PCa receiving ADT.

\section{Sexual dysfunction}

8 The adverse effects of ADT that relate to sexual dysfunction can result in a perceived loss of masculinity and

9 difficulties in the relationship dyad. Qualitative research conducted alongside a RCT which involved interviews

10 with PCa patients found that exercise reinforces masculinity and thus enhances sexual wellbeing [43].

11 Evidence from one systematic review [29] and one RCT indicates exercise can enhance sexual health following

12 PCa treatment and exercise initiated with treatment can help to maintain sexual function in men who were

13 sexually active prior to commencing ADT [44].

\section{Psychiatric disorders}

15 Depression and anxiety

16 A PCa diagnosis is a major source of life stress for most men and treatment can exacerbate this effect,

17 contributing to significant decrements in quality of life. Age, cancer stage, comorbidities, psychological

18 disposition, self-efficacy, even marital status, can impact on the nature and severity of this effect[45].

19 From a physiological perspective, exercise causes alterations to hormones (e.g. endorphin and monoamine

20 levels), corticosteroids, pro-inflammatory cytokines, growth factors and neurogenesis, impacting mood and

21 cognitive function and in this way may contribute to improvements in mental wellbeing[45]. Systematic review

22 evidence shows exercise improves quality of life (including mental health domains) in the PCa population [27,

23 46, 47].

\section{General disorders}

25 Fatigue

26 In recent years, there has been recognition of the need to address the debilitating effects of cancer related

27 fatigue. Strong evidence from eight systematic reviews [25-27, 48-52] and one RCT [53] support the effectiveness of exercise in addressing fatigue during and after treatment with ADT for PCa. Moderate intensity aerobic exercise and combined aerobic and resistance programs had significant effects, with moderate to vigorous exercise most effective.

\section{Gait disturbance}

32 Functional decline is one outcome of the ageing process and ADT for men with PCa can exacerbate this decline and result in frailty that impacts significantly on activities of daily living (ADLs) and quality of life [54]. Strong evidence from eight systematic reviews [24-28, 30, 48, 51] and two RCTs [31, 33] not elsewhere included, supports the efficacy of exercise in addressing the functional decline associated with ADT. Men with PCa undergoing ADT benefit from exercise training, demonstrating consistent, positive results in physical and muscular performance.

2. Evidence of the effectiveness of exercise in managing adverse effects or disease risks in other populations

\section{Strong evidence for the effective}

Strong evidence for the effectiveness of exercise in addressing the adverse effects of ADT for PCa is currently

limited, however evidence often exists in other populations. This is the case for the musculoskeletal adverse 
1 effect, fractures. Sherrington et al. [55] conducted a comprehensive systematic review of the effectiveness of

2 exercise interventions in preventing falls in older people (i.e., older than 60 years) living in the community and

3 found reductions in falls and fall-related fractures.

\section{$4 \quad$ Metabolic changes}

5 While results for ADT induced metabolic changes are inconclusive in the PCa population, a large meta-analysis

6 of RCTs conducted in the general population demonstrated that exercise training significantly improved CVD

7 biomarkers of lipid and lipoprotein metabolism, glucose intolerance and insulin resistance, systemic

8 inflammation, and hemostasis. The effects of exercise on cardiorespiratory fitness measures also showed that

9 people with type 2 diabetes, hypertension, hyperlipidemia or metabolic syndrome appeared to benefit more from

10 exercise. Significant modification of effects on total cholesterol and low density lipoprotein cholesterol (LDL-

11 C) were also observed for people with these conditions [56].

12 A number of systematic reviews report the importance of exercise for people with diabetes[56-59]. Resistance

13 exercise improves insulin sensitivity and glucose tolerance, while improving lean body mass and strength

14 parameters. Both resistance and aerobic exercise can assist with management of blood glucose levels, lipids,

15 blood pressure, cardiovascular risk, mortality and quality of life [56-59].

\section{Cardiac disorders}

17 The effect of exercise on cardiovascular toxicity resulting from cancer treatment is an emerging discipline [13,

18 60]. Poor cardiorespiratory fitness is associated with a higher risk of treatment toxicity, higher symptom burden

19 and increased risk of all-cause and cancer specific mortality in cancer patients, but is not recognized as a

20 traditional CVD risk [60]. The importance of vascular adaptation to exercise and the impact on cardiovascular risk in the general and CVD populations is increasingly being recognized [61, 62]. A systematic review and meta-analysis of lifestyle modification programs for patients with coronary heart disease showed that comprehensive programs reduced mortality by $34 \%$ and re-incidence and re-admission rates by $35 \%$ over follow-up of between 1-5 years [62]. Following treatment, there were significant reductions in blood pressure, total cholesterol, and smoking, as well as significant improvements in exercise behaviour and dietary habits. Treatment benefits were maintained at later follow up, with the exception of smoking, and improvements in BMI had become evident[62].

\section{Nervous system disorders}

An increasing number of studies suggest exercise has a positive effect on cognition. For example, a systematic review and meta-analysis of RCTs in community dwelling adults older than 50 years found a $29 \%$ improvement in cognitive function for at least moderate intensity exercise. Results were independent of cognitive domain or cognitive status of participants [63]. Another meta-analysis of longitudinal cohort studies of physical activity in adults $\geq 40$ years, with prevention of cognitive decline and dementia as the focus, concluded there was a case for causality [64].

Management of stroke involves consideration of a number of major modifiable risk factors such as physical inactivity [65], high cholesterol, hypertension, metabolic syndrome, diabetes, diet and nutrition, obesity and body fat distribution [65, 66], cigarette smoking, and alcohol [65]. Exercise has been shown to have a lowering effect on hypertension in several meta-analyses [67-69]. Significant modification of effects on total cholesterol and LDL-C have also been observed for people with hypertension, hyperlipidemia and metabolic syndrome in another systematic review [56]. Exercise also increases blood flow and improves the release of blood clot 
1 dissolving tissue plasminogen activator (t-PA). Stroke, a cardiovascular disease, has similar risk factors and

2 requires prevention measures like those of other cardiac disorders [36, 70, 71].

\section{Vascular disorders}

4 In four systematic reviews and meta-analyses, exercise was shown to have a lowering effect on hypertension

5 [67-69]. While included studies were heterogeneous, there was a post exercise reduction in blood pressure,

6 regardless of participant characteristics or type of exercise (aerobic and/or resistance or isometric), findings

7 supported by the American Heart Association[72]. One meta-analysis focused on isometric exercise training

8 [67]. In the other two, the lowering effect was greater when exercise was a preventive strategy in physically

9 active participants, not taking antihypertensive medication [68, 69].

10 Deep vein thrombosis is particularly prevalent amongst cancer patients and an important cause of morbidity and

11 mortality in this population [73]. One systematic review reported positive effects after exercise for patients in

12 the general population with prior or current DVT[74].

13 While there is no evidence for exercise in mitigating hot flashes for men receiving ADT, several studies have

14 noted that physically active women in the general population report fewer somatic and climacteric symptoms

15 during menopause compared to sedentary women, suggesting that physical activity is beneficial in improving

16 quality of life for this population $[75,76]$.

\section{Hepatobiliary disorders}

18 Both aerobic and resistance exercise have been shown to reduce hepatic fat content [77]. Exercise impacts on

19 fatty liver disease in a number of ways. Improved insulin resistance reduces excess delivery of free fatty acids

20 and glucose for synthesis to the liver. In the liver, exercise increases fatty acid oxidation, decreases fatty acid synthesis, and prevents mitochondrial and hepatocellular damage [77]. Two systematic reviews [78, 79] support exercise as a therapeutic strategy to improve fatty liver disease in the general population.

\section{Psychiatric disorders}

\section{Depression and anxiety}

25 A number of systematic reviews and meta-analyses have found similar effects for exercise in the general 26 population [80] and other cancer populations [81-85]. One meta-analysis of high quality exercise trials showed 27 large and significant effects, providing robust evidence for exercise in the management of depression [80]. Aerobic and mixed mode exercise were found to have large antidepressant effects across all studies when compared to no exercise controls. Supervised interventions of moderate to vigorous intensity had the largest effects and exercise supervised by professionals with relevant training such as exercise physiologists, physical educators, and physiotherapists was associated with the greatest improvements [80]. For cancer populations, several meta-analyses confirm reductions in depressive symptomology in cancer survivors following exercise interventions, mostly in those not depressed at baseline [81-85]. Cancer populations included in these meta-analyses were comprised mostly of breast cancer survivors.

\section{Exercise as medicine}

36 Evidence for each of the CTCAE ADT adverse effect sub-groups is summarised in Table 2. The source of evidence for exercise as medicine in managing the adverse effects of ADT for PCa and brief, qualitative comments on the overall quality of currently available evidence are presented. 
Table 2: Summary of evidence for exercise as medicine in managing adverse effects of ADT for PCa

\begin{tabular}{|c|c|c|c|c|}
\hline Group & $\begin{array}{l}\text { Adverse effect } \\
\text { (CTCAE) }\end{array}$ & Adverse effect sub-group & $\begin{array}{l}\text { Exercise as medicine } \\
\text { evidence }\end{array}$ & Overall quality of currently available exercise as medicine evidence \\
\hline \multirow{3}{*}{1} & \multirow{3}{*}{$\begin{array}{l}\text { Musculoskeletal } \\
\text { changes }\end{array}$} & a) Bone loss & 3 RCTs $[86,20-22]$ & $\begin{array}{l}\text { ** moderate evidence of significant improvements in bone loss; more exercise } \\
\text { RCTs and more evidence of sustained improvements needed }\end{array}$ \\
\hline & & b) Osteoporosis & $1 \mathrm{SR}[19]$ & $\begin{array}{l}\text { * exercise recommended but not alone; latest exercise RCT evidence not included } \\
\text { in this medication focussed SR }\end{array}$ \\
\hline & & c) Fracture & 0 & $\begin{array}{l}\text { * no evidence for falls or fractures; improved bone health \& physical function } \\
\text { may reduce risk of falls \& fractures }\end{array}$ \\
\hline \multirow{3}{*}{2} & \multirow{3}{*}{$\begin{array}{l}\text { Metabolic } \\
\text { changes }\end{array}$} & $\begin{array}{l}\text { a) Body composition } \\
\text { changes }\end{array}$ & $\begin{array}{l}7 \text { SRs }[24-30] \& 4 \text { RCTs } \\
{[20,22,32,33]}\end{array}$ & $\begin{array}{l}* * * \text { strong evidence for muscle strength and lean mass across } 7 \text { SRs \& } 4 \text { RCTs; } \\
\text { less consistent results for fat mass \& waist circumference }[24,25]\end{array}$ \\
\hline & & b) Metabolic syndrome & $1 \mathrm{SR}[25]$ & *inconclusive evidence for cardiometabolic risk markers from $1 \mathrm{SR}$ \\
\hline & & c) Diabetes & 0 & *no evidence to support exercise as a management strategy \\
\hline 3 & $\begin{array}{l}\text { Cardiac } \\
\text { disorders }\end{array}$ & a) Cardiovascular events & $1 \mathrm{SR}[40]$ & $\begin{array}{l}\text { *little consistent evidence, but emerging field suggesting improvements in cardiac } \\
\text { and vascular function }\end{array}$ \\
\hline \multirow{2}{*}{4} & \multirow{2}{*}{$\begin{array}{l}\text { Nervous system } \\
\text { disorders }\end{array}$} & $\begin{array}{l}\text { a) Cognitive impairment \& } \\
\text { dementia }\end{array}$ & 0 & *no conclusive evidence to support exercise as a management strategy \\
\hline & & b) Stroke & $1 \mathrm{SR}[40]$ & $\begin{array}{l}\text { *little evidence, but emerging field suggesting improvements in cardiac and } \\
\text { vascular function }\end{array}$ \\
\hline \multirow{3}{*}{5} & \multirow{3}{*}{$\begin{array}{l}\text { Vascular } \\
\text { disorders }\end{array}$} & a) Hypertension & 0 & $\begin{array}{l}\text { *no evidence, but emerging field suggesting improvements in cardiac and } \\
\text { vascular function }\end{array}$ \\
\hline & & b) Thromboembolic events & 0 & *no evidence to support exercise as a management strategy \\
\hline & & c) Hot flashes & 0 & *no evidence to support exercise as a management strategy \\
\hline 6 & $\begin{array}{l}\text { Hepatobiliary } \\
\text { disorders }\end{array}$ & a) Hepatic disorders & 0 & *no evidence to support exercise as a management strategy \\
\hline \multirow{2}{*}{7} & \multirow{2}{*}{$\begin{array}{l}\text { Reproductive } \\
\text { system } \\
\text { disorders }\end{array}$} & $\begin{array}{l}\text { a) Gynaecomastia and } \\
\text { breast pain }\end{array}$ & 0 & *no evidence to support exercise as a management strategy \\
\hline & & b) Sexual dysfunction & $1 \mathrm{SR}$ [29], 1 RCT [44] & $\begin{array}{l}* * \text { some inconsistency in evidence, but improved sexual function [29] \& } \\
\text { maintenance of sexual activity [44] reported for ADT PCa; more research needed }\end{array}$ \\
\hline 8 & $\begin{array}{l}\text { Psychiatric } \\
\text { disorders }\end{array}$ & a) Depression, anxiety & $\begin{array}{l}5 \text { SRs }[27,46,47,49 \text {, } \\
52]\end{array}$ & ** moderate evidence for HRQoL \\
\hline
\end{tabular}




\begin{tabular}{|c|c|c|c|c|}
\hline \multirow{2}{*}{9} & \multirow{2}{*}{$\begin{array}{l}\text { General } \\
\text { disorders }\end{array}$} & a) Fatigue & $\begin{array}{l}8 \text { SRs [25-27, 48-52] } \\
1 \text { RCT [53] }\end{array}$ & $* * *$ strong evidence \\
\hline & & b) Gait disturbance & $\begin{array}{l}8 \text { SRs [24-28, 30, 48, } \\
51], 2 \text { RCTs }[31,33]\end{array}$ & $* * *$ strong evidence \\
\hline
\end{tabular}

Abbreviations: CTCAE Common Terminology Criteria for Adverse Events; PCa prostate cancer; ADT androgen deprivation therapy; RCT randomised controlled trial; SR systematic review; DVT deep vein thrombosis; HRQoL health related quality of life

Notes: ${ }^{*}$ low level evidence; ${ }^{* *}$ moderate level evidence; ${ }^{* *}$ high level evidence 


\section{Discussion}

This rapid review has highlighted the evidence for the role of exercise in managing the adverse effects of ADT for PCa and identified where there is a lack of evidence (Table 2). There is strong evidence for exercise as medicine in addressing a number of the adverse effects of ADT such as reduced muscle mass and strength, fatigue and declining physical function. Moderate level evidence of the benefits of exercise was found for psychosocial effects of ADT (e.g. depression, anxiety, quality of life), particularly for supervised interventions; however, the evidence is not consistent across all of these effects [30]. Moderate level evidence also exists for bone loss and sexual dysfunction. For the remainder of the adverse effects of ADT sub groups (osteoporosis, fracture, metabolic syndrome, diabetes, cardiovascular events, cognitive impairment and dementia, stroke, hypertension, thromboembolic events, hot flashes, hepatic disorders, gynaecomastia and breast pain), evidence is non-existent or the data to support it is limited and more research is needed to address these deficiencies.

While the exercise as medicine evidence is lacking for many ADT for PCa adverse effect sub-groups, evidence in the $\mathrm{PCa}$, cancer or other clinical populations is strong and many clinical guidelines recommend exercise as a fundamental part of their clinical management [12-16]. With the exception of gynaecomastia and breast pain, there is increasing evidence to suggest that exercise has the potential to reduce and even prevent many of the adverse effects of ADT, thus improving survivorship outcomes for men with PCa. Exercise has the potential to provide an effective approach to adverse effect management, with few associated risks. It can be combined with pharmacotherapy and/or psychotherapy where required[45], however, larger, well designed studies with longer follow-up that address more adverse effects are needed to strengthen the evidence base.

Not only is exercise effective in addressing many of the adverse effects of ADT, but epidemiological evidence for PCa drawn from prospective cohort studies, also shows a moderate inverse association between physical activity and risk of advanced and fatal disease[87]. For example, outcomes from one study showed that men in the highest quintile of vigorous activity had a 77\% lower risk of advanced PCa[87]. Men with high levels of occupational activity in another study also had lower risk of advanced $\mathrm{PCa}$ [87]. Men with high levels of recreational physical activity in another study had a $31 \%$ lower risk of aggressive PCa than men who did not participate in recreational physical activity[87]. For men already diagnosed with PCa, physical activity is associated with improved survival and decreased PCa progression[10]. Vigorous activity is associated with lower risk of PCa specific mortality, both vigorous and non-vigorous activity are associated with lower risk of all-cause mortality in this population[10]. Thus, exercise is critical to health and quality of life, as well as survival, for PCa survivors.

Ideally, GPs, oncologists or urologists treating men receiving ADT for PCa would recommend exercise to manage potential adverse effects from the time of diagnosis. The ACSM advises oncologists to "Assess, Advise and Refer" so that cancer patients are connected with appropriate exercise professionals who will provide an individualised prescription[13]. The prescription could take several forms but would initially incorporate assessment and a progressive program devised and supervised by an accredited exercise physiologist or physiotherapist to assist each individual to achieve improved muscle mass and strength, cardio-respiratory fitness, fat loss and body function. Depending on the capacity of the individual PCa patient or survivor, this could be accompanied or followed by a home-based program of exercise such as cycling, walking or jogging[12, 13]. 
While considerable evidence exists to support the use of exercise as an essential part of a cancer treatment and care plan, there has been limited uptake of this approach. Research in the UK has examined the national guidelines (NICE guidelines CG175) on exercise training for men with advanced PCa to determine whether healthcare professionals were supportive of the guidelines [88] and whether they were being implemented as part of PCa care[89]. Healthcare professionals were aware of the guidelines and confused as to why no action had been taken to implement them[88]. Despite the support of healthcare professionals and men on ADT, evidencebased guidelines were not being delivered. Traditional values in oncology and the need for financial support from the government to assist translation from a hospital/clinic environment were identified as the major barriers[88]. A recent systematic review of interventions for PCa survivorship cautioned that more research is needed to examine the effectiveness and acceptability of exercise and psychosocial interventions outside clinical trials and to support translation into practice[30]. These concerns are widely recognised. The transition to widespread application clinically and post-clinically present a challenge, particularly in design and implementation, when a targeted or personalised medical approach is viewed as critical to the efficacy and safety of exercise for cancer patients $[60,90]$. There is a recognised need for prescription of exercise medicine to address variation in treatment effects, treatment intensity, patient comorbidities or fitness levels [12, 60]. This rapid review is not without its limitations. While a systematic and comprehensive search was conducted, this is not a systematic review and there may be studies that were missed in the conduct of the search. In addition, qualitative judgments of currently existing evidence were based on agreement between authors due to the heterogeneity of sources and paucity of evidence in some areas. The purpose of this review was to identify the available evidence in relation to the role of exercise in managing the adverse effects of ADT for PCa. In doing so, evidence gaps were also identified. Supplementing the evidence for the ADT PCa population with evidence from other populations supports the potential for exercise as medicine to address these evidence gaps. Future research needs to focus on the evidence gaps in relation to PCa to strengthen the current evidence base.

The use of exercise in PCa management has the potential to translate into health and economic benefits in improved quality of life and fewer complications, resulting in savings to the health care system, enhanced productivity and reduced patient and carer burden. Exercise thus has the potential to improve quality of life for this population as well as generate significant cost savings. To date, there has been no attempt to quantify the economic impact of exercise programs for men with PCa. Future research should determine the economic impact of exercise in managing the adverse effects of ADT via cost-effectiveness analysis of exercise interventions for PCa patients and survivors. Such evidence is needed to inform decision makers of the health and economic impact of exercise to support effective implementation of exercise training for PCa patients and survivors in real world settings and thus achieve research translation.

\section{Funding}

Kim Edmunds' PhD was supported by an Australian Post Graduate Awards (APA) Scholarship and an Inspiring Minds Scholarship from Edith Cowan University, WA.

\section{Conflict of Interest}

The authors declare that they have no conflicts of interest. 
References

1. Australian Institute of Health and Welfare (AIHW). Cancer in Australia: an overview 2014. Canberra: AIHW; 2014.

2. International Agency for Research on Cancer (IARC). Cancer Today: Prostate Cancer. GLOBOCAN World Health Organisation, France. 2018. http://gco.iarc.fr/today/data/factsheets/cancers/27-Prostate-fact-sheet.pdf.

3. Gordon L, Tuffaha H, James R, Keller A, Lowe A, Scuffham P et al. Estimating the healthcare costs of treating prostate cancer in Australia: a Markov modelling analysis. Urologic Oncology: Seminars and Original Investigations. 2018;36.

4. National Comprehensive Cancer Network (NCCN). Clinical Practice Guidelines in Oncology (NCCN Guidelines) Prostate Cancer NCCN, Pennsylvania. 2018.

https://www.nccn.org/professionals/physician_gls/default.aspx. Accessed December 212019.

5. Edmunds K, Tuffaha H, Galvão D, Scuffham P, Newton R. Incidence of the adverse effects of androgen deprivation therapy for prostate cancer: a systematic review. Support Care Cancer. 2020;28:2079-93. doi:10.1007/s00520-019-05255-5.

6. National Institutes of Health and National Cancer Institute. Common Terminology Criteria for Adverse Events (CTCAE) Version 5.0. 2017.

7. Vina J, Sanchis-Gomar F, Martinez-Bello V, Gomez-Cabrera M. Exercise acts as a drug; the pharmacological benefits of exercise. British Journal of Pharmacology. 2012;167:1-12.

8. Hayes S, Spence R, Galvão D, Newton R. Australian Association for Exercise and Sport Science position stand: optimising cancer outcomes through exercise. Journal of Science and Medicine in Sport. 2009;12(4):428 34.

9. Schmitz KH, Courneya KS, Matthews C, Demark-Wahnefried W, Galvão DA, Pinto BM et al. American College of Sports Medicine Roundtable on Exercise: Guidelines for Cancer Survivors. Medicine and Science in Sports and Exercise. 2010;42(7):1407-26.

10. Kenfield S, Stampfer M, Giovannucci E, Chan J. Physical activity and survival after prostate cancer diagnosis in the Health Professionals Follow-Up Study. J Clin Oncol. 2011;29:726-32.

11. Newton RU, Galvão DA. Exercise medicine for prostate cancer. Eur Rev Aging Phys Act. 2013;10:41-5. doi:10.1007/s11556-012-0114-4.

12. Hayes S, Newton R, Spence R, Galvão D. The Exercise and Sports Science Australia position statement: exercise medicine in cancer management. Journal of Science and Medicine in Sport. 2019;22(11):1175-99.

13. Campbell K, Winters-Stone K, Wiskemann J, May A, Schwartz A, Courneya K et al. Exercise Guidelines for Cancer Survivors: Consensus Statement from International Multidisciplinary Roundtable. Medicine \& Science in Sports \& Exercise. 2019;51(11):2375-90.

14. Resnick MJ, Lacchetti C, Bergman J, Hauke RJ, Hoffman KE, Kungel TM et al. Prostate cancer survivorship care guideline: American society of clinical oncology clinical practice guideline endorsement. J Clin Oncol. 2015;33(9):1078-85. doi:10.1200/JCO.2014.60.2557.

15. National Comprehensive Cancer Network (NCCN). NCCN Guidelines Version 1.2019: Survivorship. 2019. 16. National Institute for Health and Care Excellence (NICE). Prostate cancer: diagnosis and management. 2019.

17. Oxford Centre for Evidence-Based Medicine (OCEBM) Levels of Evidence Working Group. The Oxford 2011 Levels of Evidence. 2016.

18. Grossmann M, Zajac JD. Androgen deprivation therapy in men with prostate cancer: How should the side effects be monitored and treated? Clin Endocrinol (Oxf). 2011;74(3):289-93.

doi:http://dx.doi.org/10.1111/j.1365-2265.2010.03939.x.

19. Joseph JS, Lam V, Patel MI. Preventing osteoporosis in men taking androgen deprivation therapy for prostate cancer: a systematic review and meta-analysis. Eur Urol Oncol. 2019;2(5):551-61.

doi:10.1016/j.euo.2018.11.001.

20. Taaffe DR, Galvão DA, Spry N, Joseph D, Chambers SK, Gardiner RA et al. Immediate versus delayed exercise in men initiating androgen deprivation: effects on bone density and soft tissue composition. BJU Int. 2018;123(2):261-9. doi:10.1111/bju.14505.

21. Uth J, Hornstrup T, Schmidt JF, Christensen JF, Frandsen C, Christensen KB et al. Football training improves lean body mass in men with prostate cancer undergoing androgen deprivation therapy. Scandinavian Journal of Medicine and Science in Sports. 2014;24(SUPPL.1):105-12.

22. Newton RU, Galvão DA, Spry N, Joseph D, Chambers SK, Gardiner RA et al. Exercise Mode Specificity for Preserving Spine and Hip Bone Mineral Density in Prostate Cancer Patients. Medicine \& Science in Sports \& Exercise. 2018;51(4):607-14. doi:10.1249/MSS.0000000000001831. 
23. Newton RU, Jeffery E, Galvão DA, Peddle-McIntyre CJ, Spry N, Joseph D et al. Body composition, fatigue and exercise in patients with prostate cancer undergoing androgen-deprivation therapy. BJU Int.

2018;122(6):986-93. doi:10.1111/bju.14384.

24. Baumann FT, Zopf EM, Bloch W. Clinical exercise interventions in prostate cancer patients: a systematic review of randomized controlled trials. Support Care Cancer. 2012;20(2):221-33.

25. Gardner JR, Livingston PM, Fraser SF. Effects of exercise on treatment-related adverse effects for patients with prostate cancer receiving androgen-deprivation therapy: a systematic review. J Clin Oncol.

2014;32(4):335-46.

26. Hasenoehrl T, Keilani M, Sedghi Komanadj T, Mickel M, Margreiter M, Marhold M et al. The effects of resistance exercise on physical performance and health-related quality of life in prostate cancer patients: a systematic review. Support Care Cancer. 2015;23(8):2479-97. doi:10.1007/s00520-015-2782-x.

27. Bourke L, Smith D, Steed L, Hooper R, Carter A, Catto J et al. Exercise for men with prostate cancer: a systematic review and meta-analysis. European Urology. 2016;69(4):693-703.

28. Keilani M, Hasenoehrl T, Baumann L, Ristl R, Schwarz M, Marhold M et al. Effects of resistance exercise in prostate cancer patients: a meta-analysis. Support Care Cancer. 2017;25:2953-68.

29. Yunfeng G, Weiyang H, Xueyang H, Yilong H, Xin G. Exercise overcome adverse effects among prostate cancer patients receiving androgen deprivation therapy. Medicine (United States). 2017;96(27).

doi:10.1097/MD.0000000000007368.

30. Crawford-Williams F, March S, Goodwin BC, Ralph N, Galvão DA, Newton RU et al. Interventions fo prostate cancer survivorship: a systematic review of reviews. Psychooncology. 2018;27:1339-2348.

31. O’Neill RF, Haseen F, Murray LJ, O’Sullivan JM, Cantwell MM. A randomised controlled trial to evaluate the efficacy of a 6-month dietary and physical activity intervention for patients receiving androgen deprivation therapy for prostate cancer. J Cancer Surviv. 2015;9(3):431-40. doi:10.1007/s11764-014-0417-8.

32. Gilbert SE, Tew GA, Fairhurst C, Bourke L, Saxton JM, Winter EM et al. Effects of a lifestyle intervention on endothelial function in men on long-term androgen deprivation therapy for prostate cancer. British Journal of Cancer. 2016;114(4):401-8. doi:10.1038/bjc.2015.479.

33. Galvão DA, Taaffe DR, Spry N, Cormie P, Joseph D, Chambers SK et al. Exercise Preserves Physical Function in Prostate Cancer Patients with Bone Metastases. Medicine \& Science in Sports \& Exercise. 2018;50(3):393-9. doi:10.1249/MSS.0000000000001454.

34. Grossmann M, Zajac J. Management of the side effects of androgen deprivation therapy. Endocrinology and Metabolism Clinics of North America. 2011;40(4):655-71. doi:10.1016/j.ecl.2011.05.004.

35. Grossmann M, Hamilton EJ, Gilfillan C, Bolton D, Joon DL, Zajac JD. Bone and metabolic health in patients with non-metastatic prostate cancer who are receiving androgen deprivation therapy. Medical Journal of Australia. 2011;194(6):301-6.

36. Arnett D, Blumenthal R, Albert M, Buroker A, Goldberger Z, Hahn E et al. 2019 ACC/AHA Guideline on the Primary Prevention of Cardiovascular Disease: A Report of the American College of Cardiology/American Heart Association Task Force on Clinical Practice Guidelines. Circulation. 2019;140:e596-e646. doi:10.1161/ CIR.0000000000000678.

37. Colberg S, Sigal R, Yardley J, Riddell M, Dunstan D, Dempsey P et al. Physical activity/exercise and diabetes: a position statement of the American Diabetes Association. Diabetes Care. 2016;39(11):2065-79. 38. The Royal Australian College of General Practitioners \& Diabetes Australia. General practice management of type 2 diabetes 2016-2018. 2016.

39. Collins L, Mohammed N, Ahmad T, Basaria S. Androgen deprivation therapy for prostate cancer: implications for cardiometabolic clinical care. J Endocrinol Invest. 2012;35(3):332-9.

40. Beaudry R, Liang Y, Boyton S, Tucker W, Brothers R, Daniel K et al. Meta-analysis of exercise training on vascular endothelial function in cancer survivors. Integrative Cancer Therapies. 2018;17(2):192-9.

41. Ahmadi H, Daneshmund S. Androgen deprivation therapy: evidence-based management of side effects. BJU Int. 2013;111:543-8.

42. Fagerlund A, Cormio L, Palangi L, Lewin R, Di Pompeo FS, Elander A et al. Gynecomastia in patients with prostate cancer: a systematic review. PLoS ONE. 2015;10(8). doi:10.1371/journal.pone.0136094.

43. Hamilton K, Chambers SK, Legg M, Oliffe JL, Cormie P. Sexuality and exercise in men undergoing androgen deprivation therapy for prostate cancer. Support Care Cancer. 2015;23(1):133-42. doi:10.1007/s00520-014-2327-8.

44. Cormie P, Newton R, Taaffe D, Saunders, Joseph D, Akhil Hamid M et al. Exercise maintains sexual activity in men undergoing androgen suppression for prostate cancer: a randomized controlled trial. Prostate Cancer Prostatic Dis. 2013;16:170-5. doi:10.1038/pcan.2012.52.

45. Chambers S, Dunn J, Lazenby M, Clutton S, Newton R, Cormie P et al. ProsCare: A psychological care model for men with prostate cancer. Prostate Cancer Foundation of Australia (PCFA) and Griffith University, Australia; 2013. 
46. Chipperfield K, Brooker J, Fletcher J, Burney S. The impact of physical activity on psychosocial outcomes in men receiving androgen deprivation therapy for prostate cancer: a systematic review. Health Psychol. 2014;33(11):1288-97. doi:10.1037/hea0000006.

47. Bourke L, Boorjian SA, Briganti A, Klotz L, Mucci L, Resnick MJ et al. Survivorship and Improving Quality of Life in Men with Prostate Cancer. European Urology. 2015;68(3):374-83.

doi:10.1016/j.eururo.2015.04.023.

48. Keogh JWL, MacLeod RD. Body composition, physical fitness, functional performance, quality of life, and fatigue benefits of exercise for prostate cancer patients: a systematic review. Journal Pain Symptom Manage. 2012;43(1):96-110. doi:10.1016/j.jpainsymman.2011.03.006.

49. Baguley BJ, Bolam KA, Wright ORL, Skinner TL. The effect of nutrition therapy and exercise on cancerrelated fatigue and quality of life in men with prostate cancer. Nutrients. 2017;9.

50. Larkin D, Lopez V, Aromataris E. Managing cancer-related fatigue in men with prostate cancer: a systematic review of non-pharmacological interventions. Int J Nurs Pract. 2014;20(5):549-60.

doi:10.1111/ijn.12211.

51. Moe E, Chadd J, McDonagh M, Valtonen M, Horner-Johnson W, Eden K et al. Exercise Interventions for Prostate Cancer Survivors Receiving Hormone Therapy: Systematic Review. Translational Journal of the ACSM. 2017;2(1).

52. Vashistha V, Singh B, Kaura S, Prokop L, Kaushik D. The Effects of Exercise on Fatigue, Quality of Life, and Psychological Function for Men with Prostate Cancer: Systematic Review and Meta-analyses. European Urology Focus. 2016;2:284-95.

53. Taaffe DR, Newton RU, Spry N, Joseph D, Chambers SK, Gardiner RA et al. Effects of different exercise modalities on fatigue in prostate cancer patients undergoing androgen deprivation therapy: a year-long randomised controlled trial. Eur Urol. 2017;72(2):293-9. doi:10.1016/j.eururo.2017.02.019.

54. Segal R. Physical functioning for prostate health. Can Urol Assoc J. 2014;8(7-8 Supplement 5):S162-3. doi:10.5489/cuaj.2315.

55. Sherrington C, Fairhall N, Wallbank G, Tiedemann A, Michaleff Z, Howard K et al. Exercise for preventing falls in older people living in the community (Review). Cochrane Database Sys Rev. 2019;Art No.: CD012424(1). doi:10.1002/14651858.CD012424.pub2.

56. Lin X, Zhang X, Guo J, Roberts C, McKenzie S, Wu W et al. Effects of exercise training on cardiorespiratory fitness and biomarkers of cardiometabolic health: a systematic review and meta-analysis of randomized controlled trials. Journal of the American Heart Association. 2015;4(7).

57. Pan B, Ge L, Xun YQ, Chen YJ, Gao CY, Han X et al. Exercise training modalities in patients with type 2 diabetes mellitus: a systematic review and network meta-analysis. Int J Behav Nutr Phys Act. 2018;15(1):72. doi:10.1186/s12966-018-0703-3.

58. Schellenberg E, Dryden D, Vandermeer B, Ha C, Korownyk C. Lifestyle interventions for patients with and at risk for type 2 diabetes: a systematic review and meta-analysis. Ann Intern Med. 2013;159:543-51.

59. Zanuso S, Sacchetti M, Sundberg CJ, Orlando G, Benvenuti P, Balducci S. Exercise in type 2 diabetes: genetic, metabolic and neuromuscular adaptations. A review of the evidence. Br J Sports Med.

2017;51(21):1533-8. doi:10.1136/bjsports-2016-096724.

60. Scott J, Nilsen T, Gupta D, Jones L. Exercise therapy and cardiovascular toxicity in cancer. Circulation. 2018;137:1176-91.

61. Green DJ, Hopman MT, Padilla J, Laughlin MH, Thijssen DH. Vascular adaptation to exercise in humans: role of hemodynamic stimuli. Physiological reviews. 2017;97(2):495-528.

62. Janssen V, De Gucht V, Dusseldorp E, Maes S. Lifestyle modification programmes for patients with coronary heart diease: a systematic review and meta-analysis of randomized controlled trials. European Journal of Preventive Cardiology. 2013;20:620-40. doi:DOI: 10.1177/2047487312462824.

63. Northey J, Cherbuin N, Pumpa K, Smee D, Rattray B. Exercise interventions for cognitive function in adults older than 50: a systematic review with meta-analysis. Br J Sports Med. 2018;52(3):154-60.

64. Blondell S, Hammersley-Mather R, Veerman J. Does physical activity prevent cognitive decline and dementia?: a systematic review and meta-analysis of longitudinal studies. BMC Public Health. 2014;14. 65. Meschia J, Bushnell CB, Boden-Albala B, Braun L, Dawn M. Bravata D, Chaturvedi S et al. Guidelines for the primary prevention of stroke: a statement for healthcare professionals from the American Heart Association/American Stroke Association. Stroke. 2014;45:3754-832.

66. Tsivgoulis G, Safouris A, Kim D-E, Alexandrovb A. Recent advances in primary and secondary prevention of artherosclerotic stroke. Journal of Stroke. 2018;20(2):145-66.

67. Carlson D, Dieberg G, Hess N, Millar P, Smart N. Isometric exercise training for blood pressure management: a systematic review and meta-analysis. Mayo Clin Proc. 2014;89(3):327-4.

68. Carpio-Rivera E, Moncada-Jiménez J, Salazar-Rojas W, Solera-Herrera A. Acute effects of exercise on blood pressure: a meta-analytic investigation. Arq Bras Cardiol. 2016;106(5):422-33.

doi:10.5935/abc.20160064. 
69. Cornelissen V, Smart N. Exercise training for blood pressure: a systematic review and meta-analysis. Journal of the American Heart Association. 2013;2(1):e004473.

70. Nelson MR, Doust JA. Primary prevention of cardiovascular disease: new guidelines, technologies and therapies. Med J Aust. 2013;198(11):606-10. doi:10.5694/mja12.11054.

71. National Vascular Disease Prevention Alliance (NVDPA). Guidelines for the management of absolute cardiovascular disease risk. NVDPA; 2012. p. 124.

72. Brook R, Appel L, Rubenfire M, Ogedegbe G, Bisognano J, Elliott W et al. Beyond medications and diet: alternative approaches to lowering blood pressure: a scientific statement from the American Heart Association. Hypertension. 2013;61:1360-83.

73. Key N, Khorana A, Kuderer N, Bohlke K, Lee A, Arcelus J et al. Venous thromboembolism prophylaxis and treatment in patients with cancer: ASCO clinical practice guideline update. J Clin Oncol. 2019;38(5):496-520.

74. Kahn SR, Shrier I, Kearon C. Physical activity in patients with deep venous thromboembolism: a systematic review. Thrombosis Research. 2008;122:763-73.

75. Lambiase MJ, Thurston RC. Physical activity and sleep among midlife women with vasomotor symptoms. Menopause (New York, NY). 2013;20(9):946-52.

76. Moilanen J, Aalto AM, Hemminki E, Aro AR, Raitanen J, Luoto R. Prevalence of menopause symptoms and their association with lifestyle among Finnish middle-aged women. Maturitas. 2010;67:368-74.

77. van der Windt DJ, Sud V, Zhang H, Tsung A, Huang H. The effects of physical exercise on fatty liver disease. Gene Expression. 2018;18:89-101.

78. Golabi P, Locklear C, Austin P, Afdhal S, Byrns M, Gerber L et al. Effectiveness of exercise in hepatic fat mobilization in non-alcoholic fatty liver disease: Systematic review. . World Journal of Gastroenterology.

2016;22(27):6318-27.

79. Whitsett M, van Wagner L. Physical activity as a treatment of non-alcoholic fatty liver disease: A systematic review. World Journal of Hepatology. 2015;7(16):2041-52.

80. Schuch F, Vancampfort D, Richards J, Rosenbaum S, Ward P, Stubbs B. Exercise as a treatment for depression: A meta-analysis adjusting for publication bias. Journal of Psychiatric Research. 2016;77:42-51. 81. Craft L, Vaniterson E, Helenowski I, Rademaker A, Courneya K. Exercise effects on depressive symptoms in cancer survivors: a systematic review and meta-analysis. Cancer Epidemiol Biomarkers Prev. 2012;21(1):319.

82. Brown A, Huedo-Medina T, Pescatello L, Ryan S, Pescatello S, Moker E et al. The efficacy of exercise in reducing depressive symptoms among cancer survivors: a meta-analysis. PLoS ONE. 2012;7(1):e30955.

83. Mishra S, Scherer R, Geigle P, Berlanstein D, Topaloglu O, Gotay C et al. Exercise interventions on healthrelated quality of life for cancer survivors. Cochrane Database Sys Rev. 2012;8.

84. Mishra S, Scherer R, Snyder C, Geigle P, Berlanstein D, Topaloglu O. Exercise interventions on healthrelated quality of life for people with cancer during active treatment. Cochrane Database Sys Rev. 2012;8. 85. Fong D, Ho J, Hui B, Lee A, Macfarlane D, Leung S et al. Physical activity for cancer survivors: metaanalysis of randomised controlled trials. BMJ (Online). 2012;344(e70). doi:10.1136/bmj.e70.

86. Winters-Stone KM, Dobek JC, Bennett JA, Maddalozzo GF, Ryan CW, Beer TM. Skeletal response to resistance and impact training in prostate cancer survivors. Medicine and Science in Sports and Exercise. 2014;46(8):1482-8. doi:10.1249/MSS.0000000000000265.

87. Pernar CH, Ebot EM, Wilson KM, Mucci LA. The epidemiology of prostate cancer. CSH Perspectives in Medicine 2018;8.

88. Greasely R, Turner R, Collins K, Brown J, Bourke L, Rosario D. Treatment in the STAMPEDE for castrate resistant prostate cancer in the UK: ongoing challenges and underappreciated clinical problems. BMC Cancer. 2018;18:667.

89. Bourke L, Turner R, Greasley R, Sutton E, Steed L, Smith D et al. A multi-centre investigation of delivering national guidelines on exercise training for men with advanced prostate cancer undergoing androgen deprivation therapy in the UK NHS. PLoS ONE. 2018;13(7):e0197606-e. doi:10.1371/journal.pone.0197606.

90. Schmidt M, Østergren P, Cormie P, Ragle A, Sønksen J, Midtgaard J. "Kicked out into the real world": prostate cancer patients' experiences with transitioning from hospital-based supervised exercise to unsupervised exercise in the community. Support Care Cancer. 2019;27(1):199-208. 